Research Paper

\title{
Limb-bud and Heart Overexpression Inhibits the Proliferation and Migration of PC3M Cells
}

\author{
Qicai $\mathrm{Liu}^{1}{ }^{1 *}$, Ermao $\mathrm{Li}^{3}, 4^{*}$, Long Huang 3 , Minsheng Cheng ${ }^{1,2}, \mathrm{Li} \mathrm{Li}^{3 凶}$ \\ 1. Guangdong Provincial Biomedical Engineering Technology Research Center for Cardiovascular Disease; Sino-Japanese Cooperation Platform for \\ Translational Research in Heart Failure; Laboratory of Heart Center, Zhujiang Hospital, Southern Medical University, Guangzhou 510280, China; \\ 2. Department of Cardiology; Heart Center, Zhujiang Hospital, Southern Medical University; \\ 3. State Key Laboratory of Oncology in South China, Imaging Diagnosis and Interventional Center, Sun Yat-sen University Cancer Center, Guangzhou 510660, \\ China; \\ 4. Department of Urology, Minimally Invasive Surgery Center, The First Affiliated Hospital of Guangzhou Medical University, Guangzhou 510120, China. \\ * These two authors contributed equally to this paper.
}

$\triangle$ Corresponding authors: Li Li, State Key Laboratory of Oncology in South China, Imaging Diagnosis and Interventional Center, Sun Yat-sen University Cancer Center, 651" Dongfeng Road East, Guangzhou 510060, People's Republic of China; Tel. and Fax: +86-20-87342120; E-mail: li2@mail.sysu.edu.cn (L. Li). Minsheng Cheng, Laboratory of Heart Center, Zhujiang hospital, Southern Medical University, 253\# Industrial Avenue middle, Guangzhou 510280, People's Republic of China; E-mail: gzminsheng@vip.163.com (M. Cheng)

(C) Ivyspring International Publisher. This is an open access article distributed under the terms of the Creative Commons Attribution (CC BY-NC) license (https://creativecommons.org/licenses/by-nc/4.0/). See http://ivyspring.com/terms for full terms and conditions.

Received: 2017.06.07; Accepted: 2017.11.09; Published: 2018.01.01

\begin{abstract}
Background: The limb-bud and heart gene $(L B H)$ was discovered in the early 21 st century and is specifically expressed in the mouse embryonic limb and heart development. Increasing evidences have indicated that LBH not only plays an important role in embryo development, it is also closely correlated with the occurance and progression of many tumors. However, its function in prostate cancer (PCa) is still not well understood. Here, we explored the effects of LBH on the proliferation and migration of the PCa cell line PC3M.

Methods: LBH expression in tissues and cell lines of PCa was detected by immunohistochemistry and Western blotting. Lentivirus was used to transduct the LBH gene into the PC3M cells. Stable $\mathrm{LBH}$-overexpressing PC3M-LBH cells and PC3M-NC control cells were obtained via puromycin screening. Cell proliferation was examined using the 3-(4,5-dimethylthiazol-2-yl)-2, 5-diphenyltetrazolium bromide (MTT) assay. Cell cycle distribution and apoptosis rate were investigated using flow cytometry. Cell migration was studied using the Transwell assay.

Results: LBH expression level was down-regulated in 3 different PCa cell lines, especially in PC3M cells, compared with the normal prostate epithelial cells(RWPE-1). Cell lines of LBH-upregulated PC3M-LBH and PC3M-NC control were successfully constructed. Significantly increased LBH expression level and decreased cyclin D1 and cyclin E2 expression level was found in PC3M-LBH cells as compared to the PC3M-NC cells. The overexpression of LBH significantly inhibited PC3M cell proliferation in vitro and tumor growth in nude mice. $\mathrm{LBH}$ overexpression in PC3M cell, also induced cell cycle G0/G1 phase arrest and decreased the migration of PC3M cells.

Conclusions: Our results reveal that LBH expression is down-regulated in the tissue and cell lines of PCa. LBH overexpression inhibits PC3M cell proliferation and tumor growth by inducing cell cycle arrest through down-regulating cyclin D1 and cyclin E2 expression. LBH might be a therapeutic target and potential diagnostic marker in PCa.
\end{abstract}

Key words: LBH; Prostate cancer; Proliferation; Cell cycle; Migration.

\section{Introduction}

Prostate cancer (PCa) was one of the most common malignant tumors of the male urinary system [1]. In 2016, there were approximately 180,890 new PCa cases and 26,120 PCa-related deaths in the
United States [2]. Race, high-fat diet, age, and genetic predisposition $[3,4]$ are major contributing factors to malignant transformation. Most patients will eventually develop castration-resistant PCa (CRPC) 
after 12-18 months of androgen deprivation therapy [5-7]. To date, there is no effective therapy targeting CRPC. Gene therapy appears to be a potential effective biological therapy [8, 9]. An increasing amount of evidence shows that PCa formation is multifactorial, involving genetic alteration of oncogenes and tumor suppressor genes [10, 11]. Therefore, identifying these key genes, exploring their functional roles, and investigating the underlying molecular mechanisms are essential for better understanding of PCa pathogenesis.

LBH is a highly conserved, newly discovered transcription cofactor that is specifically expressed in the mouse embryonic limb and heart, and plays important roles in embryonic development [12-14]. Hassan et al. proposed that LBH is a member of the intrinsically disordered protein (IDP) family, as it exhibits a high degree of disorder, suggesting that conformational plasticity may influence LBH-dependent transcriptional activity [15]. Reiger et al. [16] found that LBH is a direct target gene of the $\mathrm{WNT} / \beta$-catenin signaling pathway, and may act as a downstream effector of canonical $\mathrm{WNT} / \beta$-catenin signaling in epithelial development and aggressive basal subtype breast cancers. A number of studies have indicated that $\mathrm{Wnt} / \beta$-catenin signaling played an important role in PCa tumorigenesis [17]. High Wnt1 and $\beta$-catenin expression have positive correlation with $\mathrm{PCa}$ bone metastases and poor prognosis, while there is no $\beta$-catenin expression in normal prostate tissue [18]. LBH is also expressed in additional embryonicand adult tissues, including the gut, brain, spleen, lung, bones, kidney, and peripheral nervous system $[13,14,19,20]$. Previously, we found the downregulation of $\mathrm{LBH}$ in nasopharyngeal carcinoma (NPC) tissue, which correlated with a more aggressive clinical course and significantly shorter disease-specific survival in patients with NPC and LBH overexpression inhibits the growth of NPC xenograft tumors in nude mice and in vitro [21]. Despite this association with NPC tumorigenesis, the functional mechanism of LBH in PCa tumorigenesis is unknown.

Therefore, in the present study, we examined the expression pattern of $\mathrm{LBH}$ in PCa tissues, and investigated the effect of $\mathrm{LBH}$ on the cell proliferation, cell cycle and migration of PCa cells. Its potential mechanism was also further clarified.

\section{Materials and Methods}

\section{Cell lines and cell culture}

The normal human prostate epithelial cell line RWPE-1 (ATCC) was cultured in keratinocyte Serum-free medium (Gbico, Grand Island, NY, USA) with $1 \%$ penicillin and streptomycin combination (KeyGen Biotech, Nanjing, China). The human PCa cell lines DU145, PC3 and PC3M (ATCC) were maintained in RPMI1640 (Gbico) supplemented with $10 \%$ fetal bovine serum (FBS, Gbico). All cells were grown in standard cell culture conditions $\left(5 \% \mathrm{CO}_{2}\right.$, $95 \%$ humidity) at $37^{\circ} \mathrm{C}$.

\section{Construction of recombinant plasmids, the production of recombinant lentivirus and establishment of stable cell lines}

Stable cell lines were established as described previously [21]. Total RNA was reverse-transcribed with SuperScript III Reverse Transcription (Invitrogen). PCR product containing CDS of LBH gene was obtained through PCR with LBH primers (forward: 5'-CCCGTGTCATCCTCACTCG-3' and reverse: 5'-CAGATGCTGGCTGGTATGACC-3') and ligated into a CMV-ORF-SV40-eGFP-IRES-Puromycin vector (Lv201). Then, the recombinant LV-LBHs or LV-NC plasmids were co-transfected with the Gag/Pol, Rev, and VSV-G vectors into the 293T cells to produce recombinant lentivirus. After lentivirus infection of the PC3M cell, LBH-overexpressing cells PC 3M-LBH and control cells PC3M-NC were obtained via 2 -week puromycin $(2 \mu \mathrm{g} / \mathrm{ml})$ screening.

\section{Immunohistochemistry}

Immnunohistochemistry staining was performed using a previously described protocol [21]. Briefly, tissue sections were incubated overnight at $4^{\circ} \mathrm{C}$ with an anti-LBH monoclonal antibody (mAb; Sigma). Then, the sections were incubated $40 \mathrm{~min}$ with biotinylated anti-rabbit secondary antibody, and another $40 \mathrm{~min}$ with the avidin-biotinylated peroxidase complex. The sections were washed with distilled water (10 $\mathrm{min})$, treated with diaminobenzidine (DAB) to visualize positive staining. The staining intensity was visually scored and stratified as follows: 0 (negative), 1 (weak), 2 (moderate), and 3 (strong).

\section{Quantitative real-time PCR (qRT-PCR)}

Total RNA was extracted with TRIzol (Invitrogen) and $1 \mu \mathrm{g}$ of total RNA was used to produce cDNA with a PrimeScript RT Reagent Kit (AK2802, Takara, Japan). qRT-PCR was performed using the SYBR Premix Ex Taq II (RR820A Takara, Japan) with primers showed in Table 1. The mRNA levels were analyzed using the comparative threshold cycle $\left(2^{-{ }^{\triangle} \mathrm{CT}}\right)$ method, and normalized by glyceridehyde-3-phosphate dehydrogenase (GAPDH).

\section{Western blotting}

Western blot analysis was conducted as previously published by our laboratory [21]. Briefly, 
$40 \mu \mathrm{g}$ protein samples were electrophorsed on a $10 \%$ SDS-PAGE gel, transferred to PVDF membrane (Millipore, Billerica, MA, USA), and then probed with primary antibodies against $\mathrm{LBH}$ (mAb, Sigma), Cyclin D1 (mAbs, CST), cyclin E2 (mAbs, CST) and GAPDH (mAbs, CST). A horseradish peroxidase-conjugate anti-mouse and anti-rabbit IgG (1:3000; ab136815, ab136817, Abcam, UK) was then added, and the secondary antibody was detected through autoradiography using enhanced chemiluminescence (ECL Plus general electric healthcare Milwaukee, WI).

Table 1. Primers used for detection of mRNA Expression by qRT-PCR

\begin{tabular}{|c|c|c|}
\hline \multirow[t]{2}{*}{ Gene } & \multicolumn{2}{|l|}{ Primer sequences } \\
\hline & Forward $\left(5^{\prime}-3^{\prime}\right)$ & Reverse $\left(5^{\prime}-3^{\prime}\right)$ \\
\hline LBH & CCTGAGGAGTTCCTGGTCC & CAGATGCTGGCTGGTATGAC \\
\hline Cyclin D1 & GCGAAGTGGAAACCATCCGC & GCGAAGTGGAAACCATCCGC \\
\hline Cyclin E2 & CGTCACTGATGGTGCTTGCAG & CACAAGGCAGCAGCAGTCAG \\
\hline GAPDH & CGCTGAGTACGTCGTGGAGTC & $\begin{array}{l}\text { CGCTGATGATCTTGAGGCTGTTG } \\
\text { TC }\end{array}$ \\
\hline
\end{tabular}

\section{Cellular Proliferation Assay}

Cells $\left(5 \times 10^{3} /\right.$ well $)$ were cultured in $100 \mu \mathrm{L}$ RPMI 1640 medium containing 10\% FBS in 96-well plates. After $24 \mathrm{~h}, 10 \mu \mathrm{L} /$ well MTT ( $5 \mathrm{mg} / \mathrm{mL}$, Sigma) was added and the cells were incubated at $37^{\circ} \mathrm{C}$ for 4 $\mathrm{h}$. The supernatant was removed, and the formazan was dissolved in DMSO $\left(100 \mu \mathrm{L} /\right.$ well, $\left.37^{\circ} \mathrm{C}, 10 \mathrm{~min}\right)$. Absorbance $(570 \mathrm{~nm})$ was read using a microplate reader (Thermo Scientific, USA) and monitored every other day for 6 days.

\section{Colony formation assay}

Cells were seeded in six-well plates at a density of 500 cells per well and cultured for 10 days. Cell colonies were fixed with $4 \%$ paraformaldehyde solution, stained with $0.1 \%$ crystal violet, and counted under an inverted microscope.

\section{Cell cycle and apoptosis analysis}

For cell cycle analysis, Cells $\left(5 \times 10^{5}\right)$ were harvested and washed twice with ice-cold PBS, fixed with cold $70 \%$ ethanol at $-20^{\circ} \mathrm{C}$ overnight, and stained with $50 \mathrm{mg} / \mathrm{mL}$ propidium iodide (Sigma) containing $5 \mathrm{mg} / \mathrm{mL}$ RNase A. For cell apoptosis assay, Cells were trypsinized and double stained with annexin V-APC and propidium iodide using an Annexin V Apoptosis Detection Kit APC (Cat: 88-8007, ebiosicence) according to the experimental procedure. The analysis of cell cycle and apoptosis was performed with a flow cytometer (Beckman-Coulter, USA).

\section{Cell migration assays}

Serum-free RPMI 1640 medium $(100 \mu \mathrm{L})$ containing $1 \times 10^{5}$ cells was added to the top chamber of $8-\mu \mathrm{m}$ pore Transwell chambers (\#3422; Corning, Cambridge, MA, USA). The bottom chamber was filled with $750 \mu \mathrm{L}$ RPMI 1640 medium containing $10 \%$ FBS. After 24-h incubation, the cells were fixed with $4 \%$ paraformaldehyde for $10 \mathrm{~min}$ and stained with $0.1 \%$ crystal violet solution for $10 \mathrm{~min}$. Cells attached to the downside of filter were observed under inverted microscopy, and nine random fields per insert were counted.

\section{Xenograft experiment}

Four-week-old male BALB/c nude mice were supplied by the Experimental Animal Center of Guangdong province, China. All mice were maintained in a specific pathogen-free facility and were randomly divided into three groups of five mice each, namely the groups. $5 \times 10^{6}$ PC $3 \mathrm{M}, \mathrm{PC} 3 \mathrm{M}-\mathrm{LBH}$ or PC3M-NC cells were injected subcutaneously into the right axillary region of each animal. Tumor size was measured twice a week with a Vernier caliper; tumor volume was calculated as follows: $0.524 \times$ (length) $\times$ (width) $)^{2}$. After 4 weeks, the mice were sacrificed, and the tumors were dissected and weighted.

\section{Statistical analysis}

Each experiment was performed in triplicate and in parallel. Data are presented as the mean \pm SD. Differences among groups were analyzed using one-way analysis of variance. All statistical analyses were performed using SPSS 18.0 software (Chicago, IL). $\mathrm{P}<0.05$ was considered statistically significant.

\section{Results}

\section{LBH expression in tissues and cell lines of PCa}

We analyzed the expression and distribution of LBH protein in PCa tissues by immunohistochemistry (Fig. 1A). LBH was strongly positively expressed in adjacent non-cancer epidermal cells of prostate cancer tissue, while lower LBH expression was observed in almost all PCa tissues, especially in high Gleason score (GS) PCa tissue. We also found the expression of LBH was down-regulated at mRNA and protein level in 3 PCa cell lines, especially in PC3M cells, as compared with normal prostate epithelial cells RWPE-1 (Fig. 1B, 1C, P<0.05). Therefore, PC3M with relatively lower LBH expression was selected for the subsequent experiments.

\section{Successful construction of PC3M-LBH cell line}

To explore the function of LBH in PCa cells, a stable LBH-upregulated PC3M-LBH cell line and a PC3M-NC control cell line were established (Figure 2A). qRT-PCR indicated that LBH in PC3M-LBH cells was overexpressed by up to $>150$ times (Fig. $2 \mathrm{~B}, \mathrm{P}<$ 
0.001), and the protein expression of LBH was concordant with the mRNA level (Fig. 2C, $\mathrm{P}<0.05$ ). These findings suggest that the stable
LBH-overexpression cell line PC3M-LBH was successfully constructed.

A

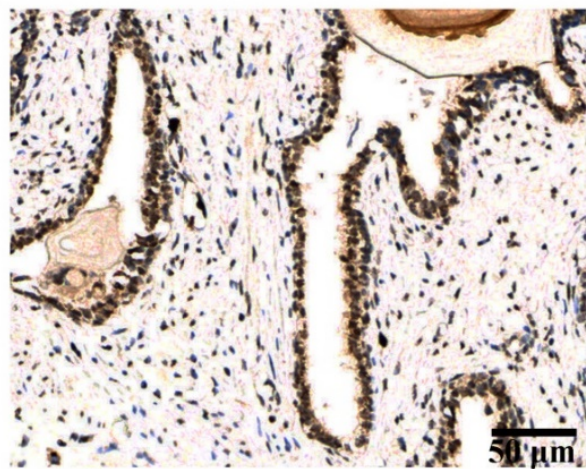
Adjacent benign tissue
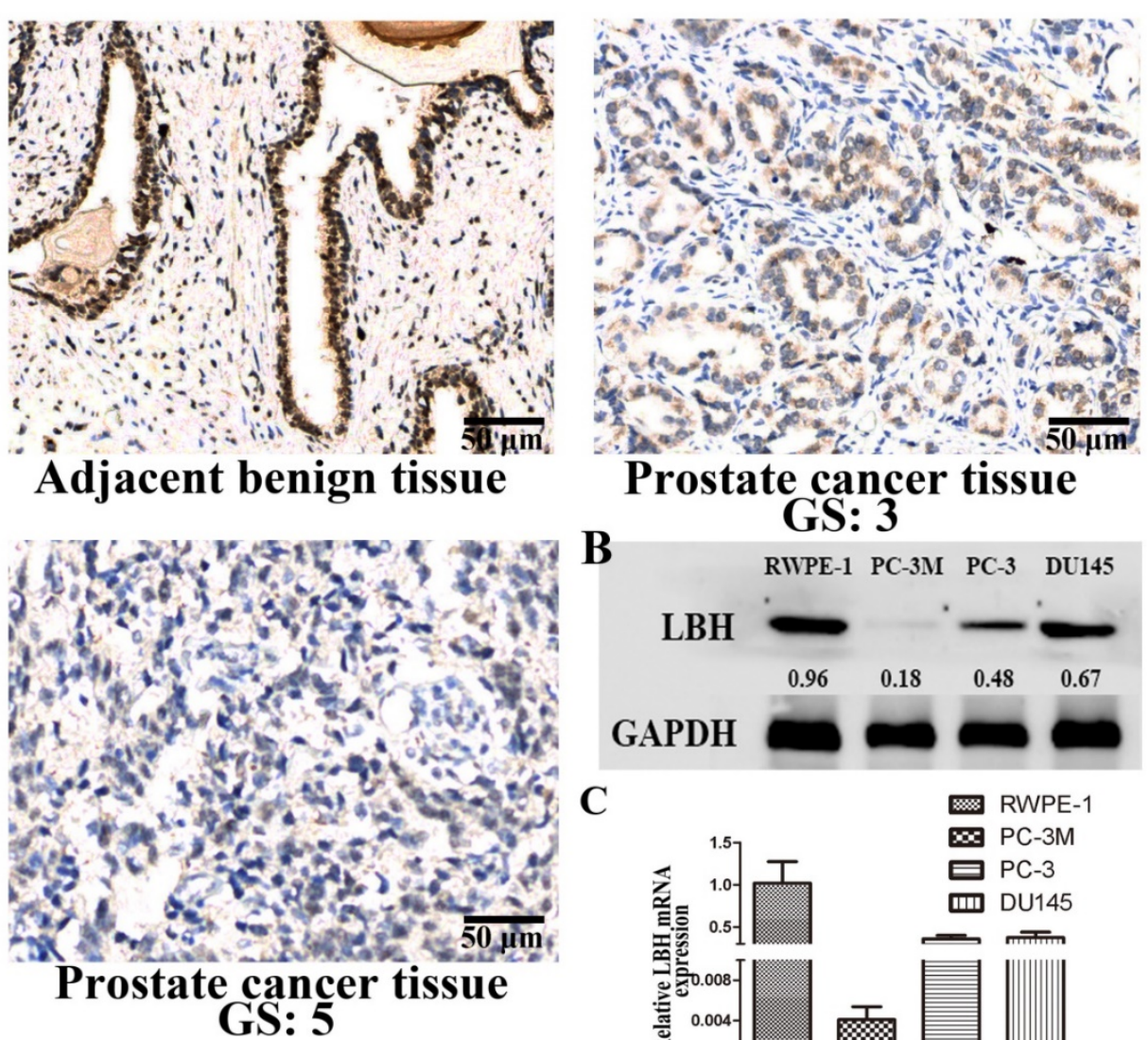

B
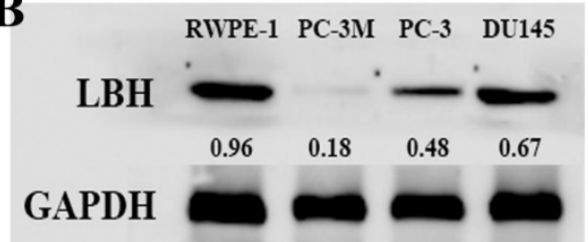

C

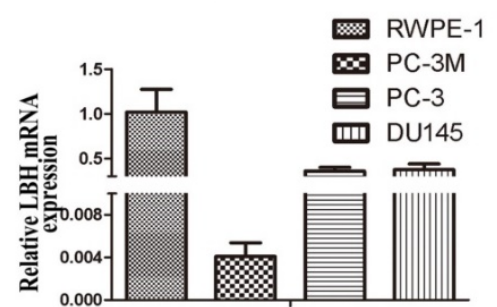

Figure 1. LBH expression in tissues and cell lines of prostate cancer. (A) Representative photograph of LBH expression in PCa tissue detected by IHC. Adjacent benign prostate non-cancer epidermal cells showed strong positive LBH staining. PCa tissue with Gleason score 3 exhibited moderately positive LBH staining. PCa tissue with Gleason score 5 tissue were negative for $\mathrm{LBH}$ staining. (B, C) The mRNA and protein expression of LBH in 3 prostate cancer cell lines;

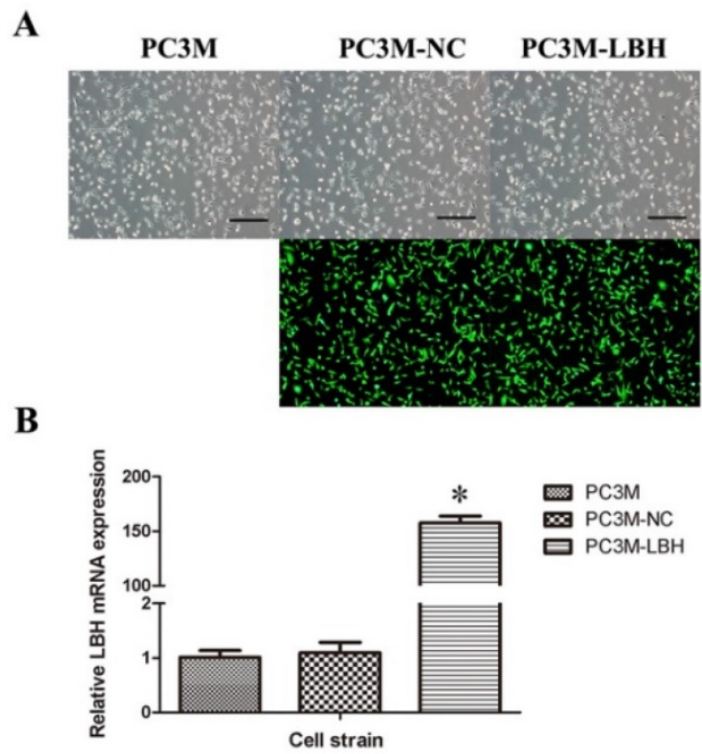

C

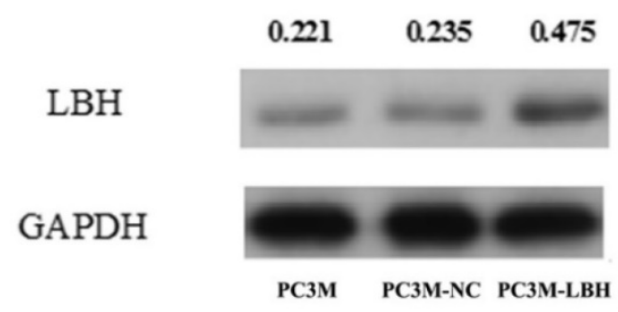

Figure 2. Successful construction of PC3M-LBH cell line. (A) Representative photograph of PC3M cells infected with lentivirus LV-NC and LV-LBH. (B, C) GRT-PCR and Western blot analysis of LBH expression in PC3M, PC3M-NC and PC3M-LBH cells. ( ${ }^{*} \mathrm{P}<0.05$, PC3M-LBH vs. PC3M-NC) 


\section{LBH inhibits the growth of PC3M cell in vitro and in vivo}

The proliferation of PC3M-LBH was inhibited by $24 \%$ compared to PC3M cells or PC3M-NC cells on day 6 (Fig. 3A, P < 0.05). The number of PC3M-LBH cell colony was significantly decreased compared to PC3M-NC cell colony (Fig. 3B, P < 0.05). We verified the results using a nude mice xenograft model in vivo. The tumor size and weight of PC3M-LBH cells were also decreased compared to that of the PC3M-NC cell (Fig. 4A, P < 0.05). IHC analysis revealed that the percentage of $\mathrm{LBH}$ positive cells was greatly increased in PC3M-LBH tumors than in PC3M-NC tumors (Fig. $4 \mathrm{~B} ; \mathrm{P}<0.05)$. These results suggest that $\mathrm{LBH}$ overexpression in PCa cells suppresses PCa growth.

\section{LBH induces $G 1$ to $S$ arrest of PC3M cells}

PC3M-LBH cells had a lower ratio of S-phase cells but a higher ratio of G1-phase cells than PC3M cells and PC3M-NC cells (Fig. 5A). The apoptosis rate of PC3M-LBH cells was no significntly change as compared with that of PC3M cells and PC3M-NC cells $(\mathrm{P}>0.05)$ (Fig. 5B). Taken together, these results suggest that $\mathrm{LBH}$ can induce G1 arrest in PC3M; however, it has no significant effect on cell apoptosis of PC3M.

\section{LBH reduces $P C 3 M$ cell migration}

The effect of $\mathrm{LBH}$ on PCa cell motility was assessed using the Transwell migration assay. The result showed LBH significantly reduced the migration of the PC3M-LBH cells as compared to the PC3M cells and the PC3M-NC cells ( $<$ < 0.001) (Fig. 6),

A

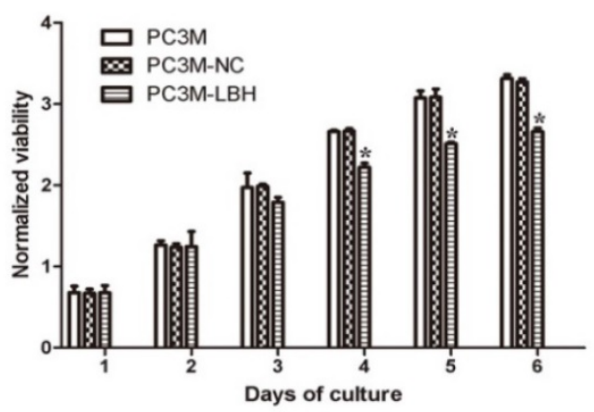

and the result suggest that $\mathrm{LBH}$ expression reduce the metastatic potential of PC3M cells effectively.

\section{Upregulated LBH inhibits the expression of cell cycle-related genes}

To determine the mechanism of cell cycle arrested by $\mathrm{LBH}$, we detected the expression of cell cycle-related genes at mRNA and protein levels. Cyclin D1 and Cyclin E2 expression was decreased in PC3M-LBH cells as compared with PC3M-NC cells (Fig. 7, P<0.05).

\section{Discussion}

In the present study, we examined the expression pattern of $\mathrm{LBH}$ in PCa tissues. $\mathrm{LBH}$ was predominantly localized in the cytoplasm and nuclear both in PCa tumor cells and normal cells, and LBH expression in PCa tissues was lower than that in the non-tumor tissues. We also found that $\mathrm{LBH}$ expression was low in the PCa cells compared with the normal RWPE-1prostate epithelial cells. These findings suggest that the loss of expression of $r \mathrm{LBH}$ may contribute to the carcinogenesis of PCa. To determine the function of $\mathrm{LBH}$ in $\mathrm{PCa}$, we constructed a stable LBH-upregulated PC3M-LBH cell clone, and found that LBH expression in the PC3M-LBH cells was upregulated by $>150$ times as compared with PC3M-NC or PC3M cells after several passages, indicating successful construction of a stable LBH-overexpression PC3M-LBH cell clone. Furthermore, LBH overexpression suppressed the PCa cell proliferation properties in vitro and tumor growth in vivo.

B

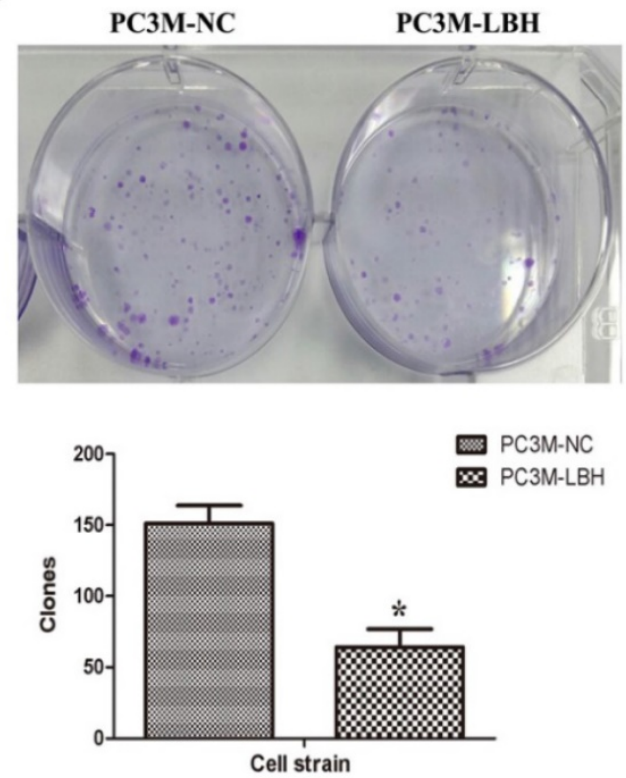

Figure 3. Effects of $\mathrm{LBH}$ on cell proliferation and colony formation. (A) $\mathrm{LBH}$ expression inhibited the proliferation of PC3M cells. (B, C) The expression of $\mathrm{LBH}$ significantly decreased the number of colony formation of PC $3 M-L B H$ cells. ( $* P<0.05, P C 3 M-L B H$ vs. PC $3 M-N C)$ 
A

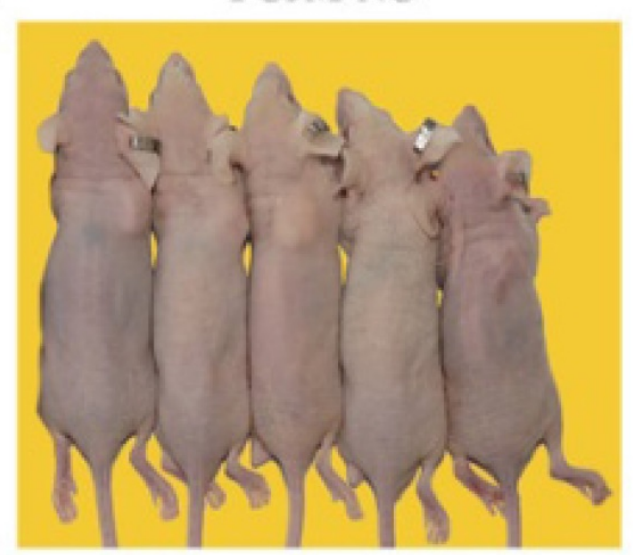

B

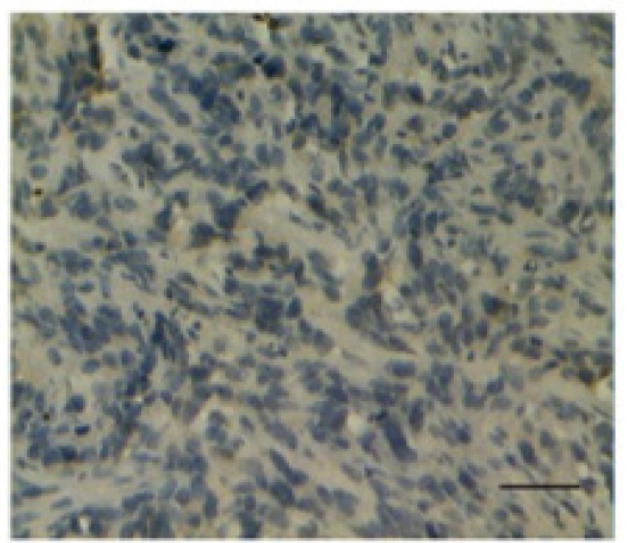

C

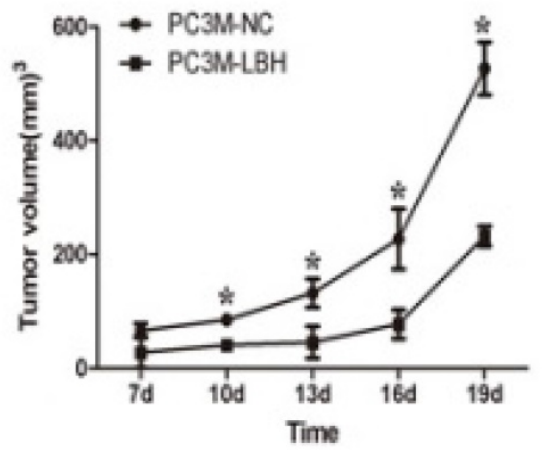

PC3M-LBH
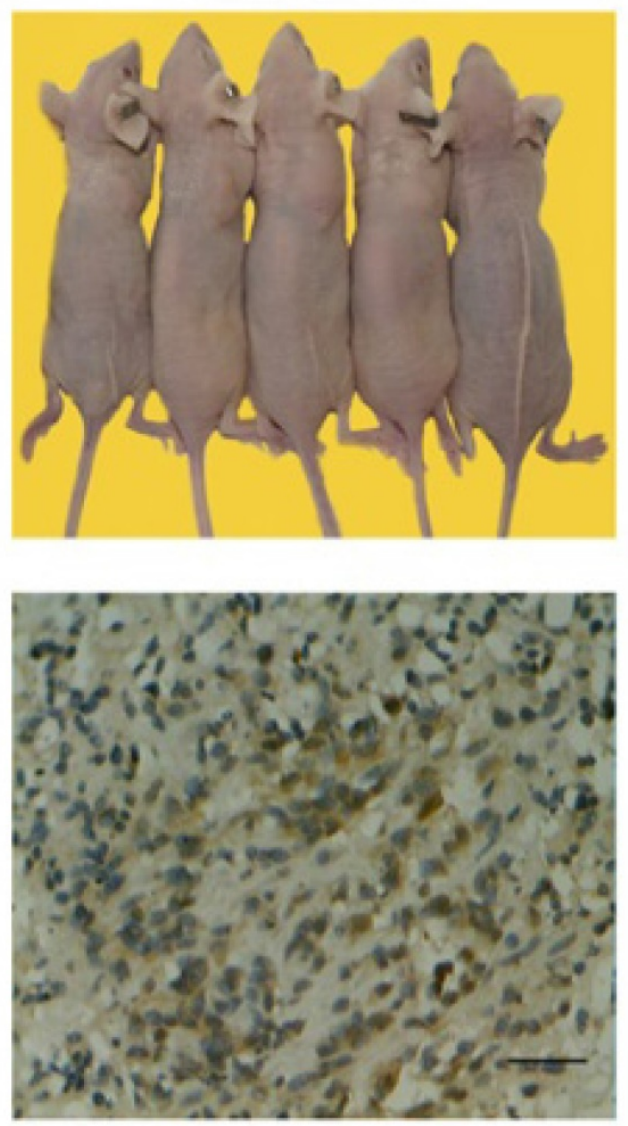

D

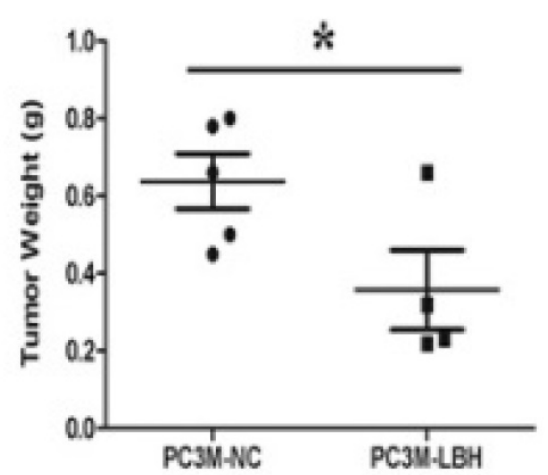

Figure 4. $\mathrm{LBH}$ inhibited tumor growth of $\mathrm{PCa}$ in vivo. (A, C, D) tumors in nude mice, tumor volume and weight of $\mathrm{PC} 3 \mathrm{M}-\mathrm{LBH}$ and $\mathrm{PC} 3 \mathrm{M}-\mathrm{NC}$ cells $(* \mathrm{P}<0.05$, PC3M-NC vs. PC3M-LBH). (B) IHC staining of LBH protein in tumor xenografts.

The potential mechanisms underlying the tumor-suppression role of LBH were also explored. The mechanism of disordered cell cycle regulation is an important cause of uncontrolled cell proliferation that leads to cancer [22]. The precise transition from G1 to $S$ phase in the cell cycle is essential for the control of eukaryotic cell proliferation, and its misregulation promotes oncogenesis [23]. We found that $\mathrm{LBH}$ overexpression in the PC3M-LBH cells significantly induced cell cycle arrest in the G1/0 phase as compared with PC3M cells and PC3M-NC cells, and there was a simultaneous, statistically significant reduction of cells in $\mathrm{S}$ phase and G2/M phase, while LBH overexpression had no statistically significant effect on the apoptosis. Furthermore, LBH inhibited the protein expression of the cell cycle-related gene cyclin D1 and cyclin E2 in PC3M cells. c-Myc is a key cell cycle regulator; many studies have found that c-Myc acts as an oncogene, playing indispensable roles in tumor formation and maintenance $[24,25]$. Cyclin D1 and Cyclin E2 are key signaling nodes that regulate $\mathrm{G} 1$ to $S$ phase transition 
$[26,27]$. These results are consistent with our previous study on NPC [21]. LBH typically induces NPC cell cycle arrest at G1/S transition, and can suppress the growth of transplanted NPC tumors in vivo. Taken together, these data indicate that LBH overexpression potently inhibits PCa cell growth by inducing cell cycle arrest in the G0/G1 phase, rather than apoptosis, which strongly suggests the role of $\mathrm{LBH}$ in $\mathrm{PCa}$ cell proliferation. The molecular mechanisms involved in the cell cycle inhibition require further investigation.
A

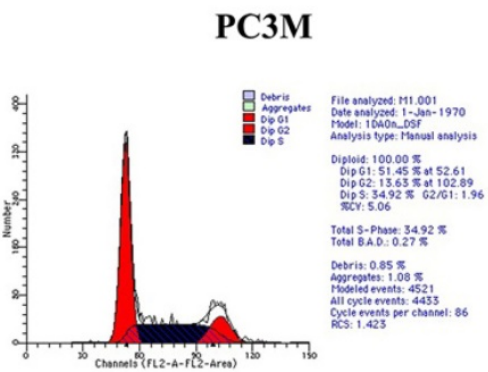

B

PC3M

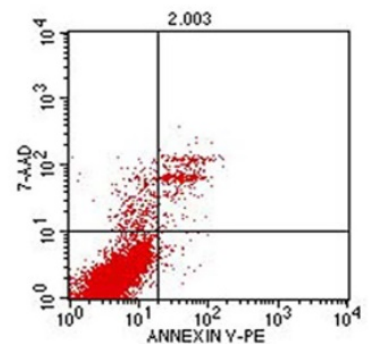

PC3M-NC

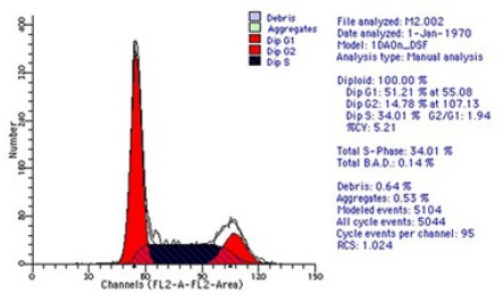

PC3M-NC

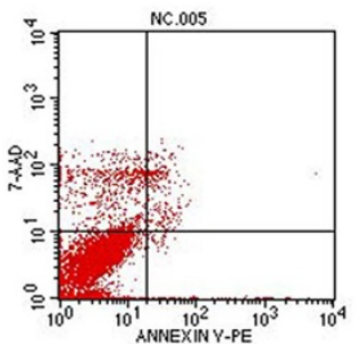

PC3M-LBH

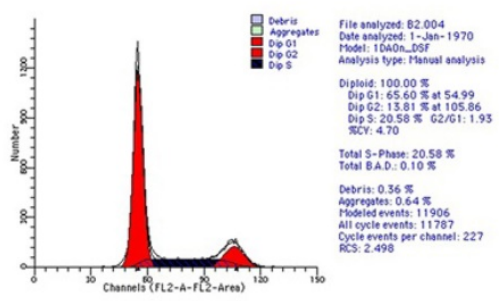

PC3M-LBH

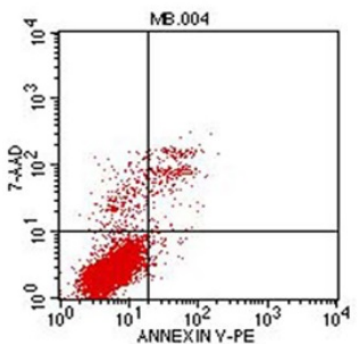

Figure 5. Effect of LBH on PCa cell cycle and apoptosis. (A) Flow cytometry analysis of cell cycle distribution. (B) Cell apoptosis detection with Flow cytometry.
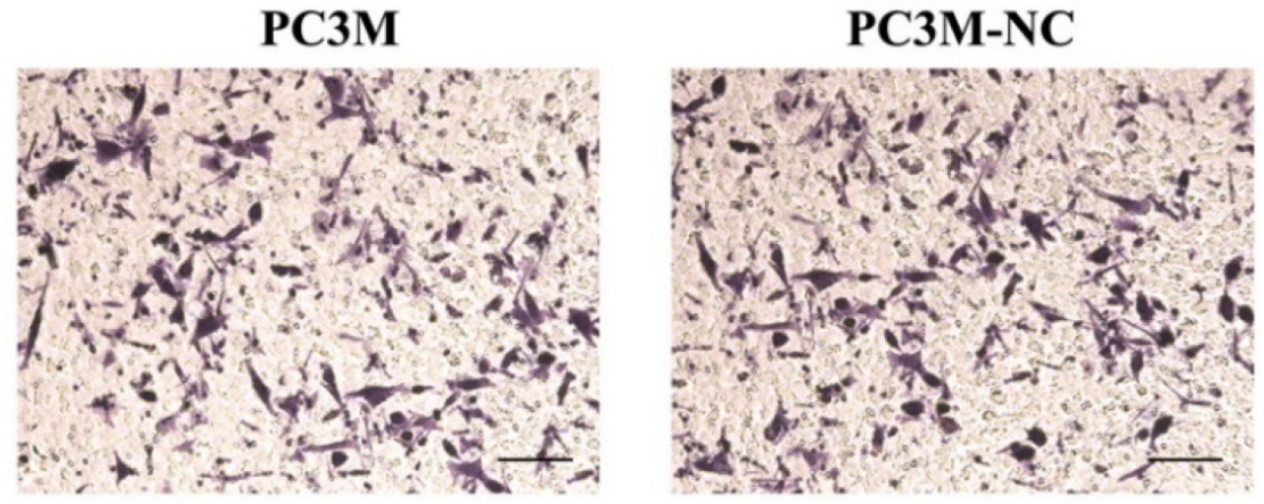

PC3M-LBH
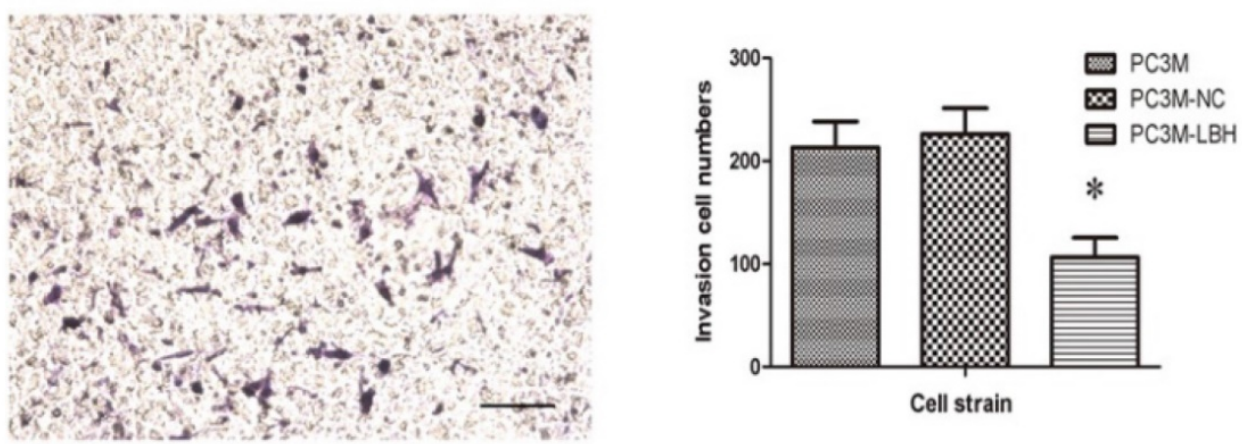

Figure 6. The effect of LBH on PCa cell migration. Migration cell numbers was decreased in PC3M-LBH as compared to PC3M and PC3M-NC. (*P < 0.05 , PC3M-LBH vs. PC3M-NC) 
A
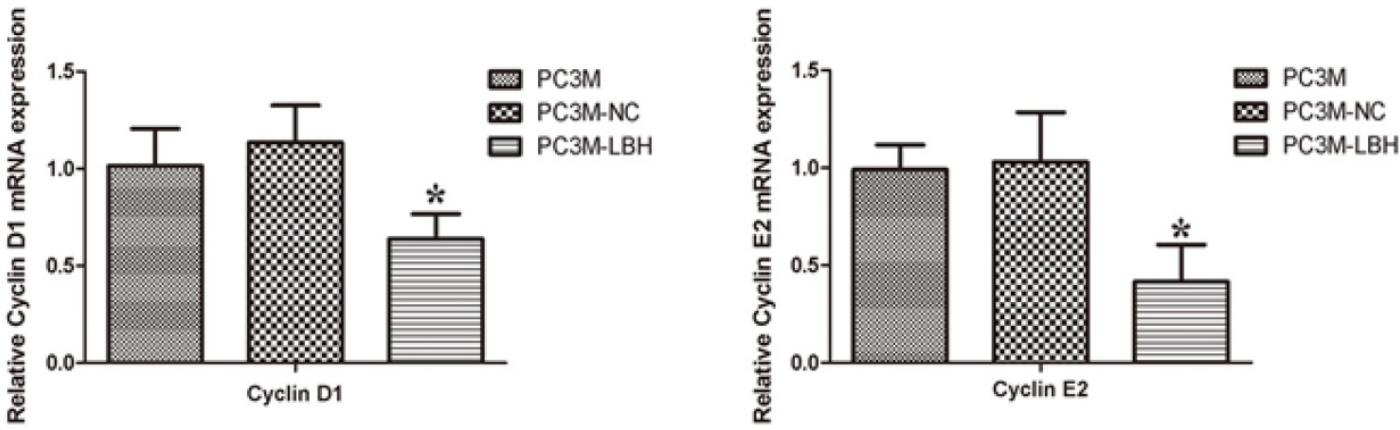

B

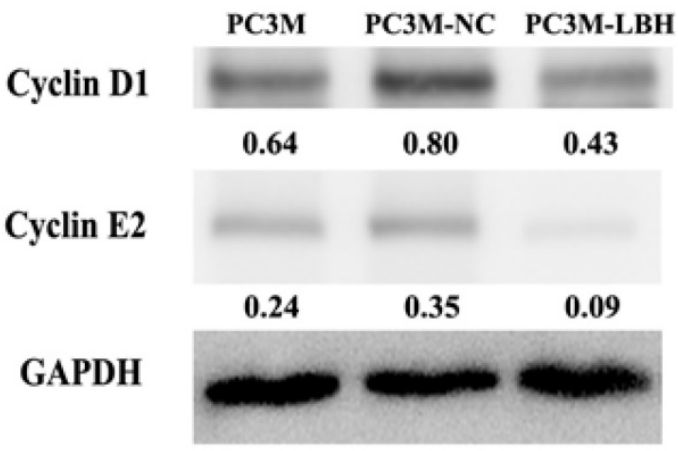

Figure 7. Effect of LBH on the expression of cell cycle-related genes. (A) qRT-PCR analysis of cyclin D1 and cyclin E2 mRNA levels; (B) western blot analysis of Cyclin D1 and cyclin E2 protein. The expression of Cyclin D1 and cyclin E2 was decreased in PC3M-LBH cells compared with PC3M-NC cells (*P $<0.05$ PC3M-LBH vs. PC3M-NC)

Taken together, the present study confirms that $\mathrm{LBH}$ inhibits the proliferation of PC3M PCa cells by downregulating the cell cycle-related gene cyclin D1 and cyclin E2, and induces cell cycle arrest in G0/G1 phase. Our studies provided new insights into the molecular mechanism involved in the regulation of PCa growth and provide novel therapeutic targets and strategies for treating PCa.

\section{Acknowledgment}

This work was financially supported by the National Natural Science Foundation of China (No. 81471711), Natural Science Foundation of Guangdong, China (No.2014A030311036), and Indstry, Education and Research Program of Guangdong (No.20130914).

\section{Competing Interests}

The authors have declared that no competing interest exists.

\section{References}

1. Center MM, Jemal A, Lortet-Tieulent J, et al. International variation in prostate cancer incidence and mortality rates. Eur Urol. 2012; 61(6):1079-1092.
2. Siegel RL, Miller KD, Jemal A. Cancer statistics, 2016. CA: A Cancer Journal for Clinicians. 2016; 66(1):7-30

3. Berezney R. The nuclear matrix: a heuristic model for investigating genomic organization and function in the cell nucleus. J Cell Biochem. 1991; 47(2):109-123.

4. Leman ES, Getzenberg RH. Nuclear structure as a source of cancer specific biomarkers. J Cell Biochem. 2008; 104(6):1988-1993.

5. Drake CG. Prostate cancer as a model for tumour immunotherapy. Nat Rev Immunol. 2010; 10(8):580-593.

6. Chen CD, Welsbie DS, Tran C, et al. Molecular determinants of resistance to antiandrogen therapy. Nat Med. 2004; 10(1):33-39.

7. Hegeman RB, Liu G, Wilding G, et al. Newer therapies in advanced prostate cancer. Clin Prostate Cancer. 2004; 3(3):150-156.

8. Sonpavde G, Thompson TC, Jain RK, et al. GLIPR1 tumor suppressor gene expressed by adenoviral vector as neoadjuvant intraprostatic injection for localized intermediate or high-risk prostate cancer preceding radical prostatectomy. Clin Cancer Res. 2011; 17(22):7174-7182.

9. Patel P, Young JG, Mautner V, et al. A phase I/II clinical trial in localized prostate cancer of an adenovirus expressing nitroreductase with CB1954 [correction of CB1984]. Mol Ther. 2009; 17(7):1292-1299.

10. Lindquist KJ, Paris PL, Hoffmann TJ, et al. Mutational Landscape of Aggressive Prostate Tumors in African American Men. Cancer Res. 2016; 76(7):1860-1868.

11. Mitchell T, Neal DE: The genomic evolution of human prostate cancer. Br J Cancer. 2015; 113(2):193-198.

12. Briegel KJ, Baldwin HS, Epstein JA, et al. Congenital heart disease reminiscent of partial trisomy $2 \mathrm{p}$ syndrome in mice transgenic for the transcription factor Lbh. Development. 2005; 132(14):3305-3316.

13. Briegel KJ, Joyner AL. Identification and characterization of Lbh, a novel conserved nuclear protein expressed during early limb and heart development. Dev Biol. 2001; 233(2):291-304.

14. Conen KL, Nishimori S, Provot S, et al. The transcriptional cofactor Lbh regulates angiogenesis and endochondral bone formation during fetal bone development. Dev Biol. 2009;333(2):348-358 
15. Al-Ali H, Rieger ME, Seldeen KL, et al. Biophysical characterization reveals structural disorder in the developmental transcriptional regulator LBH. Biochem Biophys Res Commun. 2010; 391(1):1104-1109.

16. Rieger ME, Sims AH, Coats ER, et al. The embryonic transcription cofactor LBH is a direct target of the Wnt signaling pathway in epithelial development and in aggressive basal subtype breast cancers. Mol Cell Biol. 2010; 30(17):4267-4279.

17. Logan CY, Nusse R. The Wnt signaling pathway in development and disease. Annu Rev Cell Dev Biol. 2004; 20:781-810.

18. Chen G, Shukeir N, Potti A, et al. Up-regulation of Wnt-1 and beta-catenin production in patients with advanced metastatic prostate carcinoma: potential pathogenetic and prognostic implications. Cancer. 2004; 101(6):1345-1356.

19. Gawantka V, Pollet N, Delius H, et al. Gene expression screening in Xenopus identifies molecular pathways, predicts gene function and provides a global view of embryonic patterning. Mech Dev. 1998; 77(2):95-141.

20. Paris J, Philippe M. Poly(A) metabolism and polysomal recruitment of maternal mRNAs during early Xenopus development. Dev Biol. 1990; 140(1):221-224.

21. Liu Q, Guan X, Lv J, et al. Limb-bud and Heart (LBH) functions as a tumor suppressor of nasopharyngeal carcinoma by inducing G1/S cell cycle arrest. Sci Rep. 2015; 5:7626.

22. Hanahan D, Weinberg RA. Hallmarks of cancer: the next generation. Cell. 2011; 144(5):646-674.

23. 23. Bertoli C, Skotheim JM, de Bruin RA. Control of cell cycle transcription during G1 and S phases. Nat Rev Mol Cell Biol. 2013; 14(8):518-528.

24. Calado DP, Sasaki Y, Godinho SA, et al. The cell-cycle regulator c-Myc is essential for the formation and maintenance of germinal centers. Nat Immunol. 2012; 13(11):1092-1100.

25. Taira N, Mimoto R, Kurata M, et al. DYRK2 priming phosphorylation of c-Jun and c-Myc modulates cell cycle progression in human cancer cells. J Clin Invest. 2012; 122(3):859-872.

26. Musgrove EA, Caldon CE, Barraclough J, et al. Cyclin D as a therapeutic target in cancer. Nat Rev Cancer. 2011; 11(8):558-572.

27. Muller-Tidow $\mathrm{C}$, Metzger $\mathrm{R}$, Kugler $\mathrm{K}$, et al. Cyclin E is the only cyclin-dependent kinase 2-associated cyclin that predicts metastasis and survival in early stage non-small cell lung cancer. Cancer Res. 2001; 61(2):647-653. 